\title{
Calidad de vida en docentes climatéricas de la Universidad Nacional de San Cristóbal de Huamanga
}

\section{Quality of life in Universidad Nacional de San Cristóbal de Huamanga professors during the climacteric}

Maritza Rodríguez-Lizana ${ }^{\text {1,a }}$, Vilma Zorrilla-Delgado ${ }^{1, b}$, Roaldo Pino-Anaya ${ }^{1, b}$, Walter Wilfredo Ochoa-Yupanqui 1,c

Filiación y grado académico

Universidad Nacional de San Cristóbal de

Huamanga, Ayacucho, Perú.

Doctora en Educación.

Magíster en Salud Pública.

Magíster en Docencia Universitaria.

(D) ORCID iD de Maritza Rodríguez-Lizana

https://orcid.org/0000-0003-2634-3418

10 ORCID iD de Vilma Zorrilla-Delgado https://orcid.org/0000-0002-9930-1190

(D) ORCID iD de Roaldo Pino-Anaya https://orcid.org/0000-0002-8199-3731

10 ORCID iD de Walter Ochoa-Yupanqui https://orcid.org/0000-0001-6591-4530

Contribución de los autores

MRL: conceptualización, validación, redacción

del borrador original, revisión y edición.

VZD: conceptualización e investigación.

RPA: conceptualización, administración del proyecto e investigación.

WWOY: software, validación, análisis formal, revisión y edición.

Fuentes de financiamiento

La investigación fue financiada por la Oficina de Investigación e Innovación de la Universidad $\mathrm{Na-}$ cional de San Cristóbal de Huamanga.

Conflictos de interés

Los autores declaran no tener conflictos de interés en la publicación de este artículo.

Recibido: 01-04-2021

Arbitrado por pares

Aceptado: 30-06-2021

Citar como

Rodríguez-Lizana M, Zorrilla-Delgado V, Pino-Anaya R, Ochoa-Yupanqui WW. Calidad de vida en docentes climatéricas de la Universidad Nacional de San Cristóbal de Huamanga. Rev Perú Cienc Salud. 2021; 3(3): 159-63. doi: https://doi.org/10.37711/ rpcs.2021.3.3.355

\section{RESUMEN}

Objetivo. Determinar la calidad de vida en docentes climatéricas de la Universidad Nacional de San Cristóbal de Huamanga (Perú). Métodos. Se realizó una investigación básica, descriptiva, observacional, transversal y prospectiva, con una muestra no probabilística e intencionada de 30 docentes de la UNSCH, previo consentimiento informado. Para la recolección de datos se utilizó la técnica de la encuesta y como instrumento el cuestionario Menopause Rating Scale (MRS), adaptado a Google Forms. Resultados. Las docentes climatéricas, en mayor porcentaje (38,9\%) consideraron sintomatología leve en el dominio urogenital, seguido del dominio somático y psicológico (37,5 \%); los síntomas severos se manifestaron únicamente en los dominios somático $(7,5 \%)$ y psicológico $(5,8 \%)$. Conclusiones. La escala MRS detectó un mayor deterioro del dominio psicológico y somático frente al urogenital, provocando un detrimento en la calidad de vida en docentes climatéricas de la Universidad Nacional de San Cristóbal de Huamanga.

Palabras clave: calidad de vida; climaterio; docentes (fuente: DeCS BIREME).

\section{ABSTRACT}

Objective. To determine the quality of life in Universidad Nacional San Cristóbal de Huamanga (Peru) professors during the climacteric. Methods. A basic, descriptive, observational, cross-sectional and prospective research was conducted with a non-probabilistic and intentional sample of 30 Universidad Nacional San Cristóbal de Huamanga professors, with prior informed consent. The survey technique was used for data collection and the Menopause Rating Scale (MRS) questionnaire, adapted to Google Forms, was used as a tool. Results. Professors during the climacteric, in a greater percentage (38.9\%), considered mild symptoms in the urogenital domain, followed by somatic and psychological domain (37.5\%); severe symptoms were manifested only in the somatic (7.5\%) and psychological domains (5.8\%). Conclusions. The MRS scale detected a greater deterioration of the psychological and somatic domain against the urogenital, causing a detriment in the quality of life in Universidad Nacional San Cristóbal de Huamanga professors during the climacteric.

Keywords: quality of life; climate; teachers (source: MeSH NLM). 


\section{INTRODUCCIÓN}

La calidad de vida (CV) está referida a la salud, medioambiente, entre otros ${ }^{(1)}$. Se emplea para definir el "estado de bienestar" vinculado a diversos factores; entre ellos: las relaciones sociales, estado psicológico, nivel de autonomía, las particularidades del ambiente y la salud física. Por otra parte, la calidad de vida relacionada con la salud (CVRS) ${ }^{(2)}$.

La vida de las personas implica cambios hormonales. En la mujer las glándulas sexuales determinan la vida sexual: la menarquia, el embarazo, el parto y el climaterio; muy ligados, además, a herencia, dieta, estilo de vida y actitudes culturales ${ }^{(3)}$. La menopausia es la conclusión de la menstruación por un periodo aproximado de 12 meses; antes de los 40 años, su aparición se denomina menopausia precoz ${ }^{(4,5)}$. Por su parte, el climaterio establece el paso de la vida reproductiva a la no reproductiva de la mujer; obviamente, involucra la menopausia ${ }^{(5,6)}$.

El climaterio se presenta entre los 40 a 59 años de edad ${ }^{(5,7,8)}$, iniciándose unos años antes de la menopausia (perimenopausia), y abarca algunos años después (posmenopausia) ${ }^{(5,9)}$; otros lo interpretan como el inicio del declive vital ${ }^{(7)}$, relacionados con factores biológicos y socioculturales ${ }^{(8)}$, perjudicando así la CV. Los síntomas frecuentes son vasomotores, psíquicos, atrofia urogenital, así como cambios metabólicos con riesgo de enfermedades cardiovasculares y la osteoporosis ${ }^{(10)}$. Los factores biológicos consisten en "bochornos", provocando alteraciones del sueño, emocionales ${ }^{(11)}$, cognitivos, del estado de ánimo, vaginales, urinarios, mamarios, sexuales y músculoesqueléticos ${ }^{(8)}$. La disminución de los estrógenos origina el síndrome climatérico ${ }^{(11) .}$

Se estima que para el año 2025 habrá 1,100 millones mujeres climatéricas en el mundo ${ }^{(12)}$. Esta situación requiere mejorar su estilo de vida ${ }^{(7)}$. En Latinoamérica, el $55 \%$ de las mujeres sufren esta anomalía; unos resultados que incluyen a 12 países de la región ${ }^{(13)}$, Chile y Uruguay tienen los mayores casos ${ }^{(4)}$. En el Perú, al año 2015, existía un $49,9 \%$ que para el 2050 debe llegar al climaterio ${ }^{(14)}$.

Las investigaciones sobre climaterio y $\mathrm{CV}$ en mujeres trabajadoras son contradictorias. Algunos reportan que no hay relación; otros afirman que sufren síntomas graves (2), aparentemente el trabajo disminuye la severidad de los síntomas somáticos y psicológicos, realidad que sustenta la realización del estudio; más aun considerando que las docentes universitarias alternan su trabajo con actividades domésticas.
En Lima, el Instituto Nacional Materno Perinatal (INMP) reporta desequilibrios en el peso, infecciones urinarias, modificaciones en la menstruación, vaginosis, depresión, irritación, cefalea, y otros síntomas característicos ${ }^{\left({ }^{16}\right)}$. Sin embargo, no se encuentran reportes que especifiquen la actividad laboral, por lo que nos propusimos como objetivo determinar la CV en docentes climatéricas de la Universidad Nacional de San Cristóbal de Huamanga.

\section{MÉTODOS}

\section{Tipo de estudio}

El tipo de investigación fue básica, nivel descriptivo y diseño observacional, transversal, prospectivo, de enfoque cuantitativo ${ }^{(17)}$ y se desarrolló en la Universidad Nacional de San Cristóbal de Huamanga (UNSCH), Ayacucho, Perú.

\section{Población y muestra}

La población fue de 230 docentes, mujeres, de la UNSCH con edades entre 40 a 59 años. La muestra fue seleccionada por muestreo no probabilístico intencional ${ }^{(17)}$ y estuvo conformada por un total de 30 docentes que respondieron las encuestas virtuales.

\section{Instrumentos de recolección de datos}

Para alcanzar el objetivo propuesto, se trabajó con docentes climatéricas con edades entre 40 y 59 años ${ }^{(1,4,5)}$. La variable CV se dividió en tres dominios: somático, psicológico y urogenital.

Se aplicó el cuestionario Menopause Rating Scale (MRS) ${ }^{(8,18)}$, adaptado a Google Forms, que nos permitió calcular la CV vinculada con la salud de las docentes climatéricas. Fueron evaluados once síntomas divididos en tres dominios: 1. Somático: bochornos, palpitaciones, trastornos del sueño, molestias musculares y articulares; 2. Psicológico: estado depresivo, irritabilidad, ansiedad, cansancio físico y mental; 3. Urogenital: problemas sexuales, de vejiga y sequedad vaginal. Para cada ítem, la docente asignó un puntaje de 0 a $3(0=$ ausente; $1=$ leve; 2 = moderado; 3 = severo).

\section{Procedimientos de la recolección de datos}

Los instrumentos se procesaron en un formato virtual utilizando Google Forms, publicando el enlace en los correos institucionales de dominio unsch.edu.pe, conteniendo el consentimiento informado y la explicación de la importancia del estudio.

\section{Análisis de los datos}

Los datos fueron recolectados durante los meses de junio a diciembre del 2020. La información consignada en el 
Tabla 1. Dominio somático de la CV en docentes climatéricas de la UNSCH, 2020

\begin{tabular}{|c|c|c|c|c|c|c|c|c|}
\hline \multirow{3}{*}{ Dominio somático } & \multirow{2}{*}{\multicolumn{2}{|c|}{ Ausente }} & \multicolumn{6}{|c|}{ Molestias } \\
\hline & & & \multicolumn{2}{|c|}{ Leve } & \multicolumn{2}{|c|}{ Moderada } & \multicolumn{2}{|c|}{ Severa } \\
\hline & fi & $\%$ & fi & $\%$ & fi & $\%$ & fi & $\%$ \\
\hline Bochornos & 13 & 43,3 & 11 & 36,7 & 4 & 13,3 & 2 & 6,7 \\
\hline Palpitaciones & 9 & 30,0 & 12 & 40,0 & 7 & 23,3 & 2 & 6,7 \\
\hline Trastorno de sueño & 12 & 40,0 & 10 & 33,3 & 5 & 16,7 & 3 & 10,0 \\
\hline Molestias osteomusculares & 10 & 33,3 & 12 & 40,0 & 6 & 20,0 & 2 & 6,7 \\
\hline
\end{tabular}

formulario Google Forms se descargó en un formato Excel. En dicha matriz de datos, consistente en palabras, estas fueron categorizadas a datos cuantitativos para su tratamiento con el programa estadístico SPSS versión 23.

\section{Aspectos éticos}

La investigación respetó los principios éticos de: a) Autonomía: las docentes climatéricas manifestaron su consentimiento informado, se respetó su privacidad y anonimato; la aplicación del instrumento no causó daño alguno. b) Justicia: todas las participantes fueron tratadas por igual y los resultados se emplearon exclusivamente para la investigación.

\section{RESULTADOS}

La tabla 1 muestra el dominio somático. El $43,3 \%$ de las docentes de la UNSCH afirmaron no presentar bochornos ni sudoración, el 36,7 \% presentaron síntomas leves, el $13,3 \%$ moderada y el $6,7 \%$ severa. Respecto a las palpitaciones (latidos del corazón, opresión al pecho), el $40,0 \%$ fueron leves, el 30,0 \% no presentaron molestia, el $23,3 \%$ presentaron molestias moderadas y el $6,7 \%$ severa. En trastorno del sueño (no puede dormir, duerme poco), el 40,0 \% no presentaron molestia alguna, el $33,3 \%$ presentaron leve, el $16,7 \%$ moderada y el 10,0 $\%$ severa. Las molestias osteomusculares (dolor de huesos y articulaciones, dolor reumático) fueron leves en el $40,0 \%$ de las docentes, el $33,3 \%$ no presentaron molestias, el $20,0 \%$ presentaron molestia moderada y el $2,7 \%$ severa.
Sobre el dominio psicológico, la tabla 2 evidenció que el $40,0 \%$ de las docentes no sintieron molestia alguna del estado de ánimo depresivo (se siente deprimida, decaída, triste a punto de llorar, sin ganas de vivir), seguido del $33,3 \%$ que lo fueron leve, el 20,0 \% moderada y el $6,7 \%$ severa. En cuanto a la irritabilidad (se sintió tensa, fácilmente irritable, sentimientos de ira), el 40,0 \% no presentaron molestias, el 36,7 \% leve, el $16,7 \%$ moderada y el $6,7 \%$ severa. Respecto a la ansiedad (se sintió angustiada, temerosa, inquieta, tendencia al miedo), el $46,7 \%$ no presentaron molestia alguna, seguido del 33,3\% con síntomas leves, el 13,3\% moderada y el 6,7 \% severa. Sobre el cansancio físico y mental (rindió menos, se cansó con facilidad, afrontó olvidos frecuentes, le costó concentrarse), el 46,7\% de las docentes presentaron una condición leve, el 26,7\% moderada, el $23,3 \%$ no presentaron molestia alguna y el 3,3 \% presentaron condición severa.

En la tabla 3; se presenta el dominio urogenital. El $43,3 \%$ de docentes climatéricas afrontaron problemas sexuales leves (menos deseos de compartir con su pareja, disminución en la frecuencia de relaciones sexuales, menor satisfacción sexual), el 40,0 \% no presentaron molestia y el $16,7 \%$ moderada. Respecto a problemas de vejiga, el $56,7 \%$ no presentaron molestia alguna, seguido del $33,3 \%$ que presentaron leves molestias y el $10,0 \%$ que refieren moderada. La sequedad vaginal estuvo ausente en el $50,0 \%$, el $40,0 \%$ presentaron leves problemas y el $10,0 \%$ moderada.

Tabla 2. Dominio psicológico de la CV en docentes climatéricas de la UNSCH, 2020

\begin{tabular}{|c|c|c|c|c|c|c|c|c|}
\hline \multirow{3}{*}{$\begin{array}{l}\text { Dominio } \\
\text { psicológico }\end{array}$} & \multirow{2}{*}{\multicolumn{2}{|c|}{ Ausente }} & \multicolumn{6}{|c|}{ Molestias } \\
\hline & & & \multicolumn{2}{|c|}{ Leve } & \multicolumn{2}{|c|}{ Moderada } & \multicolumn{2}{|c|}{ Severa } \\
\hline & fi & $\%$ & fi & $\%$ & fi & $\%$ & fi & $\%$ \\
\hline Depresión & 12 & 40,0 & 10 & 33,3 & 6 & 20,0 & 2 & 6,7 \\
\hline Irritabilidad & 12 & 40,0 & 11 & 36,7 & 5 & 16,7 & 2 & 6,7 \\
\hline Ansiedad & 14 & 46,7 & 10 & 33,3 & 4 & 13,3 & 2 & 6,7 \\
\hline Cansancio físico y mental & 7 & 23,3 & 14 & 46,7 & 8 & 26,7 & 1 & 3,3 \\
\hline
\end{tabular}


Tabla 3. Dominio urogenital de la CV en docentes climatéricas de la UNSCH, 2020

\begin{tabular}{|c|c|c|c|c|c|c|}
\hline \multirow{3}{*}{ Dominio urogenital } & \multirow{2}{*}{\multicolumn{2}{|c|}{ Ausente }} & \multicolumn{4}{|c|}{ Molestias } \\
\hline & & & \multicolumn{2}{|c|}{ Leve } & \multicolumn{2}{|c|}{ Moderada } \\
\hline & $\mathrm{fi}$ & $\%$ & $f i$ & $\%$ & $f i$ & $\%$ \\
\hline Problemas sexuales & 12 & 40,0 & 13 & 43,3 & 5 & 16,7 \\
\hline Problemas de vejiga & 17 & 56,7 & 10 & 33,3 & 3 & 10,0 \\
\hline Sequedad vaginal & 15 & 50,0 & 12 & 40,0 & 3 & 10,0 \\
\hline
\end{tabular}

\section{DISCUSIÓN}

El conocimiento de la sintomatología que tienen las docentes de la UNSCH coincidió con lo puntualizado por varios autores, quienes proponen que las sintomatologías del dominio somático son las más habituales, observándose sofocos y oleadas de calor ${ }^{(19)}$. Así en el dominio psicológico la mayoría refirió ausente y leve, al igual que el dominio urogenital ${ }^{(20)}$, concluyendo que todas las participantes presentaron problemas de leve a moderado y en algunos casos ausencia de los síntomas ${ }^{(7)}$, especialmente en las que llevan un estilo de vida saludable ${ }^{(6)}$. La forma de vida no saludable probablemente determine síntomas moderados y severos.

Las disfunciones sexuales son probablemente los síntomas más frecuentes en el climaterio, aunque es necesario tener en cuenta que intervienen múltiples factores, como la edad, molestias físicas y mentales, cese de las relaciones sexuales y la automedicación. En América Latina destaca la existencia de al menos un síntoma menopáusico en el $90 \%$ de las mujeres, prevaleciendo los de tipo osteomuscular, fatiga, irritabilidad y trastornos del ánimo ${ }^{(20)}$.

En el climaterio son frecuentes los ahogos $y$, principalmente, los problemas sexuales ${ }^{(7)}$, junto a la paridad ${ }^{(9)}$ aumentan el riesgo de deterioro en la $\mathrm{CV}^{(19)}$. Predominaron también los bochornos, la ansiedad, depresión, afecta la capacidad de tomar decisiones ${ }^{(20)}$, repercutiendo negativamente en el desempeño personal a nivel familiar y laboral ${ }^{(19)}$, transcendental por la función de enseñanza y otros de la docencia universitaria.

Las alteraciones psicosociales se deben a las alteraciones en los esteroides, produciendo apatía, irritación, inseguridad emocional, pérdida del libido y nerviosidad ${ }^{(15)}$.

Un aspecto muy importante, que no se puede soslayar es que el climaterio se asocia con intranquilidad, insomnio, apnea e hipopnea. Un sueño inadecuado produce efectos negativos en el estado de alerta, reduce la capacidad mental y la productividad, produciendo irritación permanente y quebrantando las relaciones familiares y sociales ${ }^{(15)}$. Para evitar estos transtornos. algunas mujeres recurren a la fitoterapia y la terapia hormonal de reemplazo ${ }^{(15)}$.

Los signos y síntomas del dominio urogenital frecuentemente son la resequedad vaginal, dispareunia, infecciones urinarias bajas o vaginales recurrentes, disuria, incontinencia urinaria y prolapsos de diversos grados; una diversidad en la sintomatología que afecta su $\mathrm{CV}^{(12)}$. La mayoría de las docentes universitarias climatéricas en la UNSCH consideró ausentes los síntomas en el dominio urogenital, algunas presentaron CV con molestias leves y moderadas, coincidiendo con otras investigaciones ${ }^{(18)}$. Contrariamente, estudios con mujeres indígenas de Perú y Colombia han demostrado que prevalecen las manifestaciones de deterioro urogenital por encima del deterioro somato-vegetativo y psicológico (20), probablemente estas diferencias se deban al estilo de vida por su condición laboral, hecho que conduce a una mejor $\mathrm{CV}^{(15)}$.

Los síntomas de la menopausia son distintos en cada mujer y lleva la impronta de las diferentes culturas. Para algunas mujeres no todos los síntomas son perjudiciales, pues hay quienes cumplen con cierta normalidad sus expectativas personales, sociolaborales y familiares; además, la satisfacción marital y el buen tratamiento durante la menopausia aumentan la CV de la mujer en esta etapa ${ }^{(9)}$.

La presencia de sintomatología severa en el dominio somático y psicológico hace pensar en manifestaciones de cansancio, irritabilidad e incremento del estado depresivo con predisposición al agravamiento después de la menopausia ${ }^{(20)}$, representando riesgos en el proceso de enseñanza-aprendizaje por el deterioro de las relaciones entre sus compañeros de trabajo y con los estudiantes.

En la sintomatología de los dominios evaluados con la escala MRS se determinó un mayor deterioro del dominio psicológico y somático frente al urogenital, provocando un detrimento en la CV en docentes climatéricas de la Universidad Nacional de San Cristóbal de Huamanga. 
Entre las limitaciones del estudio destaca que la pandemia de la Covid-19 ha limitado la toma de muestras, por lo que la investigación se basó en las respuestas obtenidas mediante el formulario Google Forms. Asimismo, se recomienda ampliar el estudio con un mayor número de muestra, teniendo en cuenta también el origen, la carrera o especialidad y el régimen de dedicación que podrían tener influencias en la CV.

\section{Agradecimientos}

A las docentes de la Universidad Nacional de San Cristóbal de Huamanga. A la Oficina de Investigación e Innovación de la Universidad Nacional de San Cristóbal de Huamanga.

\section{REFERENCIAS}

1. Monterrosa Á, Durán L, y Salguedo M. Manifestaciones menopaúsicas y calidad de vida en afrocolombianas. Valoración con escala Cervantes. latreia [Internet]. 2017 [Consultado 2021 Feb 21]; 30(4): 376-90. Disponible en: http://www.scielo.org.co/pdf/iat/v30n4/0121-0793iat-30-04-00376.pdf

2. Márquez J, Granero J, Solvas MJ, Fernández C, Rodríguez C, Parrón T. Calidad de vida en mujeres climatéricas que trabajan en el sistema sanitario y educativo. Rev Latino-Am Enfermagen [Internet]. 2011 [Consultado 2021 Feb 17]; 19(6): 9 pantallas. Disponible en: https://www. scielo.br/pdf/rlae/v19n6/es_06.pdf

3. Capote M, Segredo A, Gómez O. Climaterio y menopausia. Rev Cuba Med Gen Integr [Internet]. 2011 [Consultado 2021 Feb 21]; 27(4). Disponible en: http://scielo.sld.cu/scielo. php?script=sci_arttext\&pid=S0864-21252011000400013

4. Yabur A. La menopausia puesta al día. Gac Méd Caracas [Internet]. 2006 [Consultado 2021 Feb 21]; 114(1). Disponible en: http://www.scielo.org.ve/scielo.php?script=sci_arttext\&pid=S0367-47622006000100001\&ln$\mathrm{g}=\mathrm{es} \& \mathrm{nrm}=\mathrm{iso}$

5. De Souza R, Faria S, Silveira K, Halász F, Antunes E. El vivir de las mujeres en el climaterio: revisión sistemática de la literatura. Enferme glob [Internet]. 2012 Jan [Consultado 2021 Feb 21]; 11(25): 440-50. Disponible en: http://scielo.isciii.es/scielo.php?script=sci_arttext\&pi$d=S 1695-61412012000100025$

6. Martínez Y, Sarduy M, Rodríguez L, Rodríguez M, Iglesias B. Síntomas climatéricos según el estilo de vida en mujeres de edad mediana Climacteric symptoms and lifestyle among middle-aged women. Rev Cuba Obs Ginecol [Internet]. 2016 Jul [Consultado 2021 Feb 20]; 42(3): 295-308. Disponible en: http://scielo.sld.cu/pdf/ gin/v42n3/gin05316.pdf

7. Muñoz OA, Ruiz DA, Díaz NL, Caguana EK. Caracterización de las mujeres durante el climaterio, atendidas en una Institución del MSP de Enero a Mayo del 2020 de la ciudad de Guayaquil. J Am Heal. [Internet]. 2020 Oct 4 [Consultado 202102 4]; 3(3): 25.34. doi: https://doi. org/10.37958/jah.v3i3.48

8. Salinas $H$. Envejecimiento poblacional y unidades de climaterio. Rev chil.obstet.ginecol [Internet]. 2017 [Consultado 2021 Feb 21]; 82(3): 293-297. Disponible en: https://scielo.conicyt.cl/scielo.php?script=sci_arttext\&pi$\mathrm{d}=$ S0717-75262017000300293
9. Del Prado M, Fuenzalida A, Jara D, Figueroa R, Flores $D$, Blumel J. Evaluación de la calidad de vida en mujeres de 40 a 59 años mediante la escala MRS (Menopause Rating Scale). Rev Med Chile [Internet]. 2008 [Consultado 2021 Feb 20]; 136(12): 1511-7. Disponible en: https://scielo. conicyt.cl/pdf/rmc/v136n12/art02.pdf

10. Monsalve C, Reyes V, Parra J, Chea R. Manejo terapéutico de la sintomatología climatérica . Rev peru ginecol Obs [Internet]. 2018 [Consultado 2021 Feb 23]; 64(1): 43-50. Disponible en: http://www.scielo.org.pe/scielo.php?script=sci_arttext\&pid=S2304-51322018000100007

11. Martínez MD, Olivos M, Gómez D, Cruz P. Intervención educativa de enfermería para fomentar el autocuidado de la mujer durante el climaterio. Enfermería Univ [Internet]. 2016 Jul [Consultado 2021 Feb 21]; 13(3): 142-50. doi: https://doi.org/10.1016/j.reu.2016.04.001

12. Garrido F, Arraztoa J. El desafío clínico en el manejo de las pacientes climatéricas. Rev chil Obs ginecol [Internet]. 2018 Nov 1 [Consultado 2021 Feb 21]; 83(5): 442-3. Disponible en: https://scielo.conicyt.cl/scielo.php?scrip$\mathrm{t}=$ sci_arttext\&pid=S0717-75262018000500442

13. Menopausia. La nación. 2008 [Internet] [Consultado 2021 Feb 21]. Disponible en: https://www.nacion.com/ tecnologia/mayoria-de-mujeres-de-america-latina-empeora-calidad-de-vida-en-menopausia/KTPOZLDEUBFZTB63WAIOKPVRJA/story/

14. Instituto Nacional de Estadística e Informática. Perú [Internet] [Consultado 2021 Feb 21]. Disponible en: https:// www.inei.gob.pe/prensa/noticias/al-30-de-junio-de2015el-peru-tiene-31-millones-151-mil-643-habitantes-8500/

15. Couto D, Nápoles D. Aspectos sociopsicológicos del climaterio y la menopausia. MEDISAN [Internet]. 2014 [Consultado 2021 Feb 21]; 18(10): 1398. Disponible en: https://www.redalyc.org/articulo.oa?id=368445168011

16. Esquía J, Zárate N. Relación entre el nivel de conocimiento y la actitud frente al climaterio en mujeres atendidas en el Instituto Nacional Materno Perinatal de Lima, octubre 2017 [Internet] Lima: Universidad Norbert Wiener; 2018 [citado 2021 Feb 21]. Disponible en: http://repositorio.uwiener.edu.pe/handle/123456789/1476

17. Hernández R, Fernández C, Baptista P. Metodología de la Investigación [Internet]. $6^{\mathrm{a}}$ ed. México: Editorial Mexicana; 2014 [Consultado 2021 Feb 7]. Disponible en: https:// www.uca.ac.cr/wp-content/uploads/2017/10/Investigacion.pdf

18. Ayala F, Rodríguez $M$, Izaguirre $H$, Ayala R, Quiñones $L$, Ayala D, et al. Instrumentos utilizados para medir la calidad de vida relacionada con la salud durante el climaterio. Rev Peru Investig Matern Perinat. 2016; 5(2): 55-65. Disponible en: https://investigacionmaternoperinatal. inmp.gob.pe/index.php/rpinmp/article/view/66/67

19. Mercado M, Monterrosa Á y Duran L. Evaluación de la calidad de vida en climatéricas con la Escala Cervantes: Influencia de la etnia. Rev peru ginecol Obs [Internet]. 2018 [Consultado 2021 Feb 17]; 64(1): 13-25. Disponible en: http://www.scielo.org.pe/scielo.php?script=sci_arttext\&pid=S2304-51322018000100003

20. Navarro D. Una nueva dimensión de los síntomas vasomotores. Rev Cuba Med Gen Integr [Internet]. 2018 [Consultado 2021 Feb 24]; 34(4). Disponible en: http://www. revmgi.sld.cu/index.php/mgi/article/view/997/215 